\title{
Specimen Quality of Transanal Total Mesorectal Excision (TaTME)
}

\section{Soterios George Panousopoulos*, Panayiotis Lazarides, Georgios Panousis, Doukakis Paradellis, Nikolaos Boltsis and Constantinos Mavrantonis}

$6^{\text {th }}$ Surgical Department, Hygeia Hospital, Athens, Greece

*Corresponding Author: Soterios George Panousopoulos, 6th Surgical Department, Hygeia Hospital, Athens, Greece.
Received: April 30, 2021

Published: May 17, 2021

(C) All rights are reserved by Soterios George Panousopoulos., et al.

\section{Abstract}

Introduction: Total mesorectal excision of the rectum (TME), has been the gold standard of rectal cancer treatment since its introduction in the 1980s, greatly improving oncologic outcomes for rectal cancer patients. Minimally invasive adapatations of TME have proved to be non-inferior to the open technique. For tumors of the distal third of the rectum, however, TME continues to present significant difficulty. The advent of transanal surgery has led to Transanal Total Mesorectal Excision (TaTME), in an effort to better facilitate complete excision, while preserving the desired oncologic results.

Aim: In this study we present our experience with TaTME in 52 cases of rectal cancer patients, who were treated at our department. Patients and Methods: Between March 2018 and December 2020, 52 patients underwent TaTME for rectal cancer, performed by a single surgeon and surgical team. Pathology reports were compared to those of 48 patients who underwent laparoscopic TaSE TME by the same team between 2012 and 2019. Circumferential Resection Margin (CRM), and completeness of TME, were considered.

Results: Combined, in the TaTME group, "complete" and "nearly complete" TME specimens were documented in 92.3\% of cases. In the TaSE group, the combined "complete" and "nearly complete" specimens were $89.6 \%$ (no statistical significance- $p=0.804844$ ). In the CRM involvement investigation, pathology reported 46 cases (88.5\%) of free CRM, and 6 cases (11.5\%) of involved CRM in the TaTME group $(n=52)$. In the TaSE group $(n=48)$, there were 41 cases $(85.4 \%)$ of free CRM and 7 cases $(14.6 \%)$ of involved CRM (no statistical significance- $\mathrm{p}=0.678986)$.

Conclusion: Our experience with TaTME shows that it is an acceptable TME procedure, delivering excellent results as far as operative quality is concerned. Although hampered by a steep learning curve, it seems that TaTME is safe and efficient enough when performed in a specialized setting, and at least non-inferior to other TME approaches as far as specimen quality is concerned.

Keywords: TaTME; Transanal TME; Rectal Cancer; TME Specimen Grading; TME; Total Mesorectal Excision

\section{Introduction}

Total mesorectal excision of the rectum (TME), has been the gold standard of rectal cancer treatment since its introduction in the 1980s [1]. Oncologic outcomes for rectal cancer patients have greatly improved since [2]. Minimally invasive adapatations of TME have proved to be non-inferior to the open technique, while offering less morbidity and hospital stay. For tumors of the distal third of the rectum, however, TME continues to present significant difficulty due to the anatomy of the pelvis, even when a laparoscopic or robotic approach are used [3-8]. The advent of transanal surgery has led to Transanal Total Mesorectal Excision (TaTME), in an effort to facilitate complete excision, while preserving the desired oncologic results [9-12]. 


\section{Aim of the Study}

In this study we present our experience with TaTME in 52 cases of rectal cancer patients, who were treated at our department.

\section{Patients and Methods}

Between March 2018 and December 2020, 52 patients underwent TaTME for rectal cancer, performed by a single surgeon and surgical team. Pathology reports were compared to those of $48 \mathrm{pa}$ tients who underwent laparoscopic TaSE TME by the same team between 2012 and 2019. The 52 TaTME patients with low rectal cancer were of mean age 66 years, predominantly male $63 \%$ vs $37 \%$ ), and $78.8 \%$ had undergone pre-operative chemo-radiation. The operation was converted in 3 cases. Mean hospital stay was 6.5 days. Mean operative time was 330 minutes. The TaSE patient group were of mean age 64 years, predominantly male $(58 \%$ vs $42 \%$ ) and $70.8 \%$ had undergone pre-operative chemo-radiation. There was 1 conversion and mean hospital stay was 5.5 days in this group.

Our TaTME approach begins with transanal dissection of the rectum and mesorectum up to the level of the peritoneal recess, then laparoscopic mobilization of the abdominal rectum, sigmoid colon, descending colon, splenic flexure, and distal transverse colon. During this procedure, the Inferior Mesenteric Artery (IMA) is ligated approximately $1 \mathrm{~cm}$ from its origin at the aorta, and the Inferior Mesenteric Vein (IMV) is ligated at the ligament of Treitz. A medial-to-lateral approach is employed. We use Indocyanine Green (ICG) fluoroscopy to determine proximal colonic stump blood supply, after selecting the level of division and ligating the marginal artery. The colon is divided through a small Pfannenstiel incision and the specimen is retrieved. The colonic stump is then returned to the abdomen and guided through the pelvic resection plane out of the anus. A handsewn coloanal anastomosis is fashioned.

Pathology reports were collected and compared to pathology reports of rectal cancer patients with distal rectal cancer subjected to laparoscopic sphincter-saving transanal specimen extraction operation (TaSE) by the same surgeon and surgical team. TaSE was selected for comparison because it is a laparoscopic TME adaptation for low rectal cancers, which is the tumor location for which one would employ TaTME. Circumferential resection margin (CRM), and completeness of TME, were considered. CRM measures the distance of cancer spread into the mesorectum and evaluates the le- sion closest to the resected mesorectum's outside diameter [13]. If this distance clears $1 \mathrm{~mm}$ it is considered a free margin. Completeness of TME is measured by evaluating the bulk and smoothness of the mesorectum, the presence, size and depth of defects in the mesorectum, the presence of coning, and whether the muscularis propria is visible at any point. Specimens are classified in one of three categories: complete, nearly complete, or incomplete [14,15].

\section{Results}

In the TaTME group ( $\mathrm{n}=52)$, we recorded TME specimen grading of "complete" in 42 cases (80.8\%), "nearly complete" in 6 cases (11.5\%) and "incomplete" in 4 cases (7.7\%). Combined, in the TaTME group, "complete" and "nearly complete" TME specimens were documented in $92.3 \%$ of cases. The grading of the TME specimens in the TaSE group ( $\mathrm{n}=48)$ were "complete" in 38 cases $(79.2 \%)$, "nearly complete" in 5 cases (10.4\%) and "incomplete" in 5 cases (10.4\%). In the TaSE group, the combined "complete" and "nearly complete" specimens were $89.6 \%$. There is no statistical significance $(p=0.804844)$.

In the CRM involvement investigation between groups, pathology reported 46 cases (88.5\%) of a distance of $1 \mathrm{~mm}$ or greater, and 6 cases (11.5\%) of involved CRM or distance of less than 1 $m m$ in the TaTME group $(n=52)$. In the TaSE group $(n=48)$, there were 41 cases (85.4\%) of free CRM by $1 \mathrm{~mm}$ or more, and 7 cases (14.6\%) of involved CRM or distance of less than $1 \mathrm{~mm}$. There is no statistical significance $(\mathrm{p}=0.678986)$.

\section{Discussion and Conclusion}

TME continues to be the epitome of rectal cancer surgery. While diverse operative approach options exist with open, laparoscopic and robotic surgery offered, the anatomical barriers to a good TME operation are still in place and continue to present a challenge for rectal surgeons the world over. It is -more than ever- a team effort to confront rectal cancer, and as the contribution of a multidisciplinary team is adopted, it is also evident that outcomes are closely related to the quality of the operation performed, as that quality is measured by examination of the delivered specimen.

In this series we strive to decipher whether TaTME is safe and efficient with regard to the produced specimens, compared to more established and accepted TME procedures for low rectal cancer. Although it will require large randomized series with deep follow-up 
to answer that definitely, preliminary short-term series such as this can give insight to whether it is acceptable to continue to explore TaTME for these patients.

At first view, our experience with TaTME shows that it is an acceptable TME procedure, delivering excellent results as far as operative quality is concerned. Although hampered by a steep learning curve, making it difficult to incorporate in all hospitals, it seems that TaTME is safe and efficient enough when performed in a specialized setting, and at least non-inferior to other TME approaches as far as specimen quality is concerned.

\section{Bibliography}

1. Heald R., et al. "The mesorectum in rectal cancer surgerythe clue to pelvic recurrence?" British Journal of Surgery 69 (1982): 613-616.

2. Heald R. "The 'Holy Plane' of rectal surgery". Journal of the Royal Society of Medicine 81 (1988): 503-508.

3. Jayne D., et al. "Effect of robotic-assisted vs conventional laparoscopic surgery on risk of conversion to open laparotomy among patients undergoing resection for rectal cancer: the ROLARR randomized clinical trial". The Journal of the American Medical Association 318 (2017): 1569-1580.

4. Stevenson A., et al. "Disease-free survival and local recurrence after laparoscopic-assisted resection or open resection for rectal cancer: the australasian laparoscopic cancer of the rectum randomized clinical trial". Annals of Surgery 269 (2019): 596-602.

5. Fleshman J., et al. "Disease-free survival and local recurrence for laparoscopic resection compared with open resection of stage II to III rectal cancer: follow-up results of the ACOSOG Z6051 randomized controlled trial". Annals of Surgery 269 (2019): 589-595.

6. Fleshman J., et al. "Effect of laparoscopic-assisted resection vs open resection of stage II or III rectal cancer on pathologic outcomes: the ACOSOG Z6051 randomized clinical trial”. The Journal of the American Medical Association 314 (2015): 13461355.

7. Van Der Pas M., et al. "Laparoscopic versus open surgery for rectal cancer (COLOR II): short-term outcomes of a randomised, phase 3 trial”. The Lancet Oncology 14 (2013): 210218.
8. Jeong S., et al. "Open versus laparoscopic surgery for mid-rectal or low-rectal cancer after neoadjuvant chemoradiotherapy (COREAN trial): survival outcomes of an open-label, non-inferiority, randomised controlled trial". The Lancet Oncology 15 (2014): 767-774.

9. Sylla P., et al. "NOTES transanal rectal cancer resection using transanal endoscopic microsurgery and laparoscopic assistance". Surgical Endoscopy 24 (2010): 1205-1210.

10. Veltcamp Helbach M., et al. "Transanal total mesorectal excision for rectal carcinoma: short-term outcomes and experience after 80 cases". Surgical Endoscopy 30 (2016): 464-470.

11. Burke J., et al. "Transanal total mesorectal excision for rectal cancer: early outcomes in 50 consecutive patients". Colorectal Disease 18 (2016): 570-577.

12. Chen C., et al. "Transanal total mesorectal excision versus laparoscopic surgery for rectal cancer receiving neoadjuvant chemoradiation: a matched case-control study". Annals of Surgical Oncology 23 (2016): 1169-1176.

13. Quirke P., et al. "Local recurrence of rectal adenocarcinoma due to inadequate surgical resection. Histopathological study of lateral tumour spread and surgical excision". Lancet 2 (1986): 996-999.

14. Campa-Thompson M., et al. "Pathologic processing of the total mesorectal excision". Clinics in Colon and Rectal Surgery 28.1 (2015): 43-52.

15. Song SB., et al. "The quality of total mesorectal excision specimen: A review of its macroscopic assessment and prognostic significance". Chronic Diseases and Translational Medicine 4.1 (2018): 51-58.

\section{Volume 4 Issue 6 June 2021 (C) All rights are reserved by Soterios George Panousopoulos., et al.}

